\title{
Modeling and Simulation of a Vector-Controlled Synchronous Machine in Motoring and Generating Mode
}

\author{
Novica A. Losic \\ HONEYWELL, ON, L5L 3S6. Canada
}

\begin{abstract}
A vector-controlled permanent-magnet (PM) synchronous machine has been modeled and simulated in both the motoring and generating mode of operation. The control provides for the maximized torque and, therefore, maximum efficiency during motoring, and for a controlled power, tight voltage regulation in the presence of load changes, and minimum bus voltage ripple at the output of an $a c-d c$ converter during generation. A threephase phase-locked loop is used to provide the unity vector information in both the sensor-based and sensorless scheme. The results of the simulation are presented, including modeling details and simulation parameters.
\end{abstract}

Index Terms - Vector-based synchronous machine control, 3-phase phase-locked loops.

\section{INTRODUCTION}

Although synchronous machines with a wound rotor field are used in higher power rating applications, the advances in permanent-magnet (PM) materials result in an increased use of PM machines in applications up to several tens of kilowatts. Due to the nature of torque production mechanisms, the PM machines used in the motoring mode are usually referred to as "brushless $d c$ " motors. Indeed, it can be shown [1] that for either a PM or a wound-rotor synchronous machine used in the motoring mode, the electro-mechanical torque, $T_{e m}$, is produced in a manner analogous to that of a $d c$ machine (1).

$\mathrm{T}_{\mathrm{em}}=\mathrm{K}_{\mathrm{T}} \mathrm{I}_{\mathrm{s}} \sin \delta$

In (1), $\delta$ is the angle between the phasor of the flux, $\Phi_{\mathrm{sa}}$, in the phase- $a$ of the stator (due to the stator current whose phasor is $\mathbf{I}_{\mathbf{a}}$ ), and the phasor of the rotor/field flux in the same phase, $\Phi_{\mathrm{ra}}$ (due to either the PM or the wound-rotor field); $I_{s}$ is the peak of the stator phase current; $\mathrm{K}_{\mathrm{T}}$ denotes a machine/torque constant wherein (for a 3-phase motor) $K_{T}=$ (3/4)p $\omega_{3} N_{s} \Phi_{f}$, where $p$ is the number of machine poles, $\omega_{\mathrm{s}}=2 \pi \mathrm{f}_{\mathrm{V}}$ is the radial (synchronous) frequency of the voltage applied to the stator windings, $N_{\mathrm{v}}$ is the number of turns in each phase of the stator windings, and $\Phi_{\mathrm{f}}$ is the peak, i.e. the amplitude/length of the flux phasor $\Phi_{f}$. In the Norton representation of the machine equivalent circuit, neglecting stator winding resistance. the torque/power angle, an angle between the generated voltage / back-emf and the terminal voltage. is equal to the angle between $I_{a}$ and a phasor of the current of the Norton source [2-4]. The analogy with a $d c$ motor is seen in evaluating (1) in the case of a phasor diagram of the synchronous machine having $\delta=90^{\circ}$, shown in Fig. 1, which results in the $d c$ motor relationship

$\mathrm{T}_{\mathrm{em}}=\mathrm{K}_{\mathrm{T}} \mathrm{I}_{\mathrm{s}}$

It is because of the straightforward torque control. implied by (2), that the synchronous machine is electronically commutated to maintain $\delta$ equal to $90^{\circ}$, which effectively reduces to the so-called decoupling, i.e. to enforcing the $90^{\circ}$ phase difference between the rotor field and the filed due to the stator currents. At the same time, (2) implies the maximum torque production and, consequently, the maximum efficiency, i.e. the maximum electrical power supplied to the machine gets converted into the mechanical power, $P_{\mathrm{em}}$, available at its shaft, at a given rotor speed $\omega_{\mathrm{r}}=(\mathrm{p} / 2) \omega_{\mathrm{r}}$, as $\mathrm{P}_{\mathrm{em}}=\mathrm{T}_{\mathrm{em}} \omega_{\mathrm{r}}(2 / \mathrm{p})$.

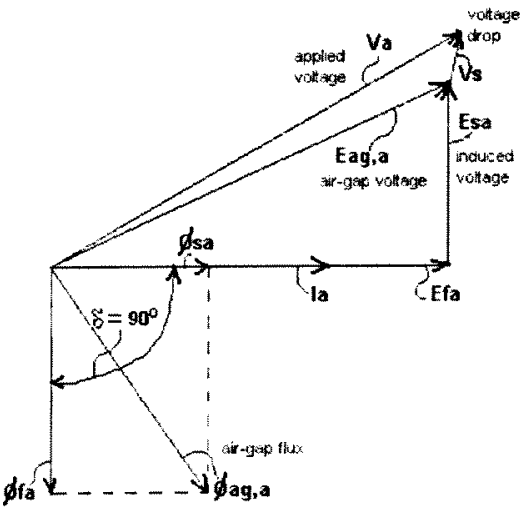

Fig. I Phasor diagram of a decoupled synchronous machine

From the phasor diagram of Fig. 1, it is seen that by enforcing the field decoupling, the stator current phasor $\mathbf{I}_{\mathrm{a}}$ gets alligned with the phasor of the back emf (in the case of the motoring mode), that is with the phasor of the generated emf (in case of the generating mode) in the corresponding phase, $\mathbf{E}_{\mathrm{fa}}$, maximizing the generated power (in case of the generating mode), as between the two (between the mechanical power, $P_{\mathrm{cm}}$, and the electrical power, $P_{e}$ ), for a 3-phase machine and neglecting both the mechanical and electrical losses, there exists the relationship below [1].

$P_{\mathrm{sm}}=\mathrm{P}_{\mathrm{e}}=3 \mathrm{E}_{\mathrm{fa}_{\mathrm{a}}} \mathrm{I}_{\mathrm{d}} \cos [\delta-(\pi / 2)]$ 
It is, therefore, of interest to use the vector control to maintain the field decoupling $\left(\delta=90^{\circ}\right)$ in the motoring mode, and to control the power in the generating mode of operation of a synchronous machine.

In the former case, the control yields the maximum torque production, as evidenced by (1), maximizing the machine efficiency for given electro-mechanical losses; in the latter it results in the control of the generated power, as implied by (3).

While, depending on the control criteria, there exists a vast number of schemes for realizing the synchronous machine control, a fraction of which is in [6-13], the approach evaluated here is based on the criterion of maximum torque-per-unit-current i.e. maximum efficiency, and on the control of the generated power, using the same basic control blocks in realizing the vector control in the two operating modes (the motoring and generating mode) of a PM synchronous machine rated at $80 \mathrm{~kW}$ of continuous power, and used in an aerospace application.

A computer model of the machine control in both modes of operation is presented, followed by the simulation results and conclusions.

\section{THE MOTORING MODE}

In the motoring mode, the control is based on providing the filed-decoupled operation, illustrated by the phasor diagram of Fig. 1, for maximum torque per unit current, i.e. maximum efficiency, whereby the block diagram of Fig. 2 is used.

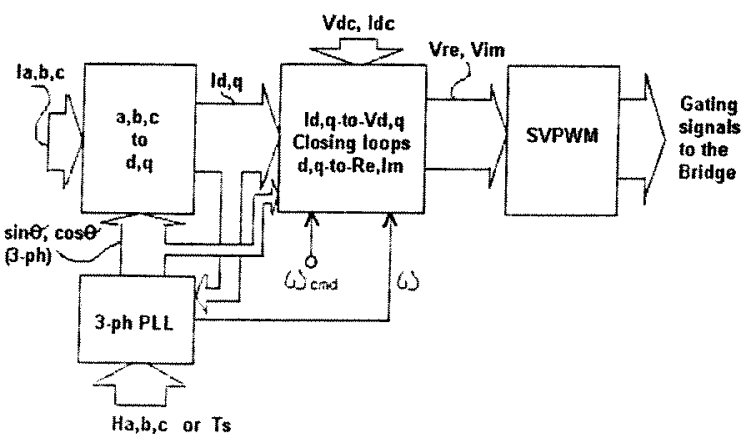

Fig. 2 Block diagram of the control in motoring mode

In Fig.2, the sensed machine current is transformed into the synchronous frame using an improved 3-phase PLL, described in [2]. wherein the PLL can use either shaft-sensor signals. $\mathrm{H}_{\text {a.h.c }}$, or receive the sampling time information, $T_{1}$, and the synchronous-frame information, $\mathbf{I}_{\mathrm{d}, 4}$, in a sensorless case, to generate the unity vector waveforms $\sin \theta \& \cos \theta$, where the phase angle $\theta$ tracks the phase of either $\mathrm{H}_{\mathrm{a}}$ or $\mathrm{I}_{\mathrm{a}}$. respectively. The $d . q$ current vector is used to provide the feedback information to close the control loops in which the $d$ axis current reference is obtained from an outer loop in which a command/reference angular speed $\omega_{\mathrm{cmd}}\left(\omega_{\mathrm{ref}}\right)$ is compared to the feedback angular speed information, $\omega=\mathrm{d} \theta / \mathrm{dt}$, supplied by the PLL (or machine model), while the $q$-axis reference is forced to zero, as required by the field-decoupling operation of Fig. 1. The result of the two inner loops processing the $\mathbf{I}_{\mathbf{d}, \mathbf{q}}$ vector is the $\mathbf{V}_{\mathrm{d}, \mathbf{q}}$ vector which, together with the unity vector, $\mathrm{e}^{\mathrm{j} \theta}$, supplied by the PLL, is used to produce the stationaryframe voltage vector, $\mathbf{V}_{\text {re,im }}\left(\mathbf{V}_{\text {Real,Imag }}\right)$. A 3-phase (time-domain) version of the $\mathbf{V}_{r e, i m}$ vector is used in the space-vector pulse-width modulation (SVPWM) block to produce the gating signals to drive the active switches in the inverter bridge. The $d c$ bus voltage/current information $\left(V_{\mathrm{dc}}, I_{\mathrm{dc}}\right)$ is used to limit the power to the machine.

While the modeling and simulation of other blocks of Fig. 2 has been referred to in $[14,15]$, Fig. 3 provides details of the model of the block of Fig. 2 used for closing the loops, the $d, q$ current-to-voltage transformation, and the $\mathbf{V}_{\mathrm{d}, \mathbf{q}}-\mathbf{V}_{\mathrm{re}, \mathrm{im}}$ transformation.

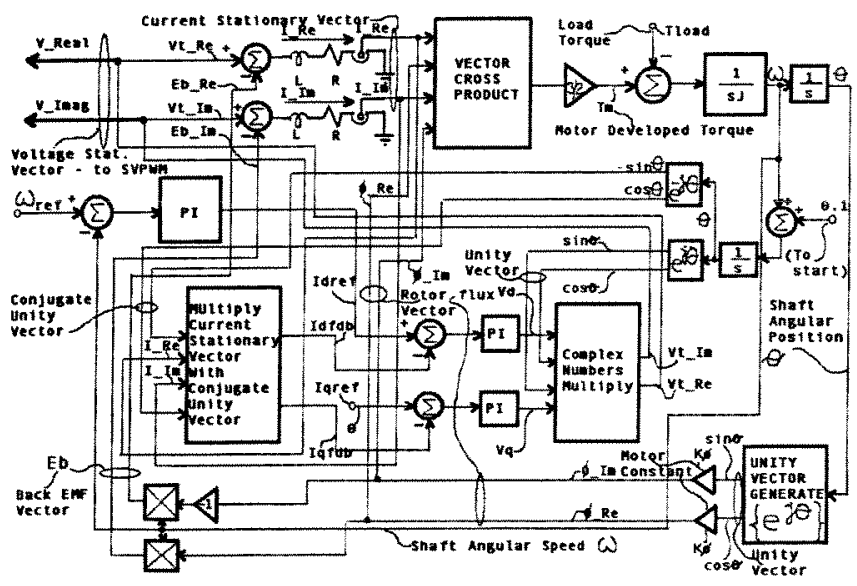

Fig. 3 Modeling the loop closure and the $d . q-R e, I m$ current-to-voltage transformation in motoring mode

While the object of the control (the controlled variable) are the three-phase currents $\mathbf{I}_{\text {a.b.c }}$ (shown in Fig. 2), the commanded speed profile is followed in the outermost loop of Fig. 3. The angular shaft position of the machine (represented with the electro-mechanical model enabling closing the loops), and/or the electrical angle/phase of the three-phase currents are used to provide additional feedback to close (some of) the inner feedback loops. Whether the sensor-based or sensorless scheme is used. the unity vector sine/cosine signals of Fig. 3 are used to provide the necessary 
reference for the complex-number/vector-frame transformations shown. As mentioned, the control enforces field decoupling in the machine, i.e. it enforces that the stator current (stationary) vector and the associated flux vector are in phase with the back emf vector, and $90^{\circ}$ ahead of the rotor flux vector. This enforces maximum torque production (consequently maximizing the efficiency) as its mechanisms are based, as shown in Fig. 3, on the cross product of the current vector and the rotor flux vector, involving the sine of the angle between them. The control is achieved by appropriately switching the inverter bridge devices to provide the desired phasing of the three-phase currents delivered to the machine relative to its shaft angular position.

\section{THE Generating MODE}

In the generating mode, in which the synchronous machine feeds a $d c$ bus through an inverter operated in the active rectifier mode, the control is based on controlling the generated power so it does not exceed a prescribed value, and providing a tight $d c$ bus voltage regulation in the presence of load changes and minimum bus voltage ripple.

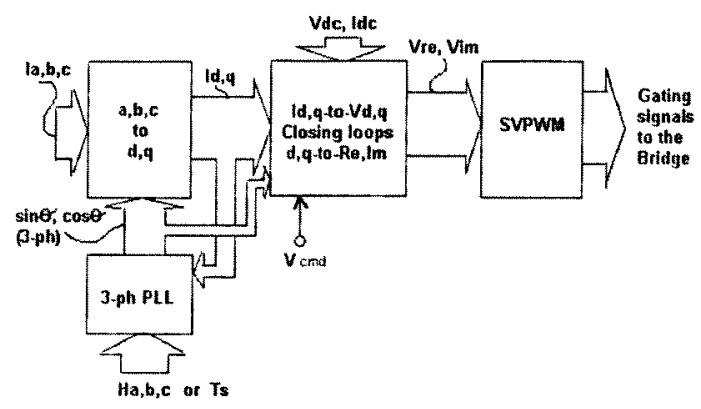

Fig. 4 Block diagram of the control in generating mode

The block diagram of Fig. 4 is used. Similarly to the case of Fig. 2, in Fig. 4 either a sensor-based or sensorless control can be utilized, with the same operations in blocks other than the block used for the loop closures and the $d . q-R e . I m$ current-to-voltage transformation, which maintains the required $d c$ bus voltage in the outermost loop, as commanded/ referenced by $\mathrm{V}_{\text {cmd }}\left(\mathrm{V}_{\text {bussef }}\right)$.

The block is depicted in Fig. 5, in which the angular shaft position of the machine, $\theta$ (obtained from the shaft speed), and/or the electrical angle/phase of the three-phase currents are used to provide additional feedback information, i.e. to close (some of) the inner feedback loops. Again. whether the sensor-based or sensorless scheme is used, the unity vector sine/cosine signals of Fig. 4 are used to provide the necessary reference for the complex-number/vector-frame transformations shown.

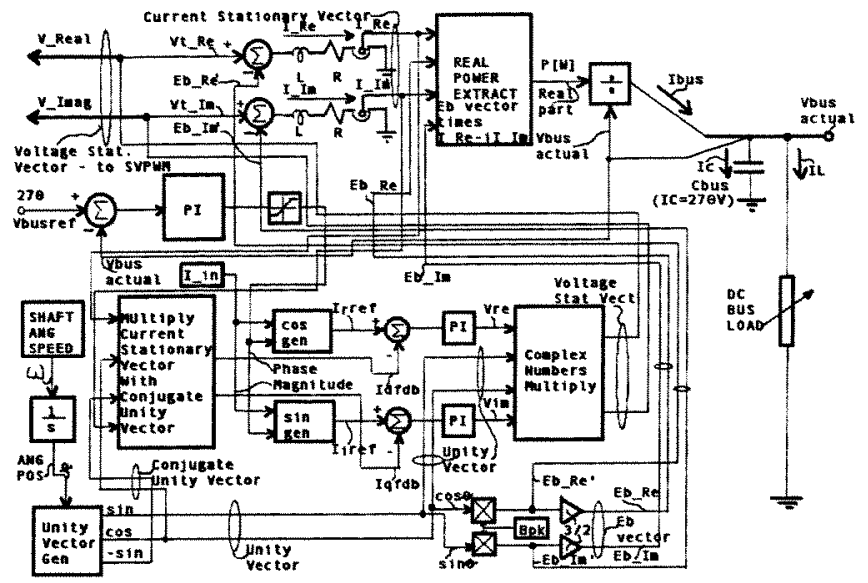

Fig. 5 Modeling the loop closure and the $d, q-R e . I m$ current-to-voltage transformation in generating mode

The angle between the stator current vector (i.e. the associated stator flux vector) and the generated emf vector is controlled. Bringing the stator current vector in phase with the generated emf vector (equivalent to feeding a zero phase input to the sin gen block of Fig. 5 ), would yield the maximum average/active/real power, because the mechanisms of the power production are based, as implied in Fig. 5, on the dot product of the stator current vector and the generated emf vector, involving the cosine of the angle between them. Namely, by definition, and as shown in the model of Fig. 5, the active/real power is extracted as a real part of the result of multiplication of the voltage vector by the conjugate of the current vector, but it can be shown that a dot product of two two-dimensional vectors, which are the vectors dealt with here, is equivalent to that.

On the other side, since the angle $\delta$ of Fig. $I$ is the angle between the rotor flux phasor, $\boldsymbol{\Phi}_{\mathrm{fa}}$, and the stator current phasor, $\mathbf{I}_{\mathrm{a}}$ (i.e. the stator field phasor, $\boldsymbol{\Phi}_{\mathrm{sa}}$ ), and since this angle is forced to $90^{\circ}$ when the current phasor, $\mathbf{I}_{\mathrm{a}}$, is brought into phase with the generated emf phasor, $\mathbf{E}_{\mathfrak{f a}}\left(\mathbf{E}_{\mathrm{b}}\right)$, the above described mechanisms of maximum electical power production in the generating mode coincide with the maximum value of the result obtained from (3) for the field decoupled operation $\left(\delta=90^{\circ}\right)$ of a synchronous machine.

From Fig. 5, in order to control the amount of the generated power, the $d, q$ current vector is used to provide the feedback information to close the control loops in which the $d$-axis current reference is obtained from a commanded magnitude, $I$ in, and the cosine of a 
phase obtained as a result of the processing provided by the ( $d c$ bus) outermost loop, while the $q$-axis current reference is obtained from $I_{-}$in and the sine of the phase.

The result of the two inner loops, which process the $\mathbf{I}_{\mathbf{d}, \mathbf{q}}$ vector, is the $\mathbf{V}_{\mathrm{d}, \mathbf{q}}$ vector $\left(\mathbf{V}_{\mathrm{re}, \mathrm{im}}\right)$, which, together with the unity vector, $\mathrm{e}^{\mathrm{j} \theta}$, supplied by the PLL, is used to produce the stationary-frame voltage vector, $\mathbf{V}_{\text {Real,Imag }}$.

Again, a 3-phase (time-domain) version of the $V_{\text {Real,Imag }}$ vector is used in the space-vector pulse-width modulation (SVPWM) block to produce the gating signals to drive the active switches in the inverter bridge operated in the rectifier mode, so as to control the conduction angle of the bridge devices as a function of the actual $d c$ bus voltage, which, in turn, is a function of load and contains ripple.

The $d c$ bus voltage/current information $\left(\mathrm{V}_{\mathrm{dc}}, \mathrm{I}_{\mathrm{dc}}\right)$ is used for closing the voltage and current loop, respectively.

\section{THE SimULATION RESULTS}

In evaluating the models of Figs. 3 and 5, the Saber simulator was used.

Fig. 6 shows the developed torque, angular position and speed during motor start, for the underdamped and optimally damped control cases.

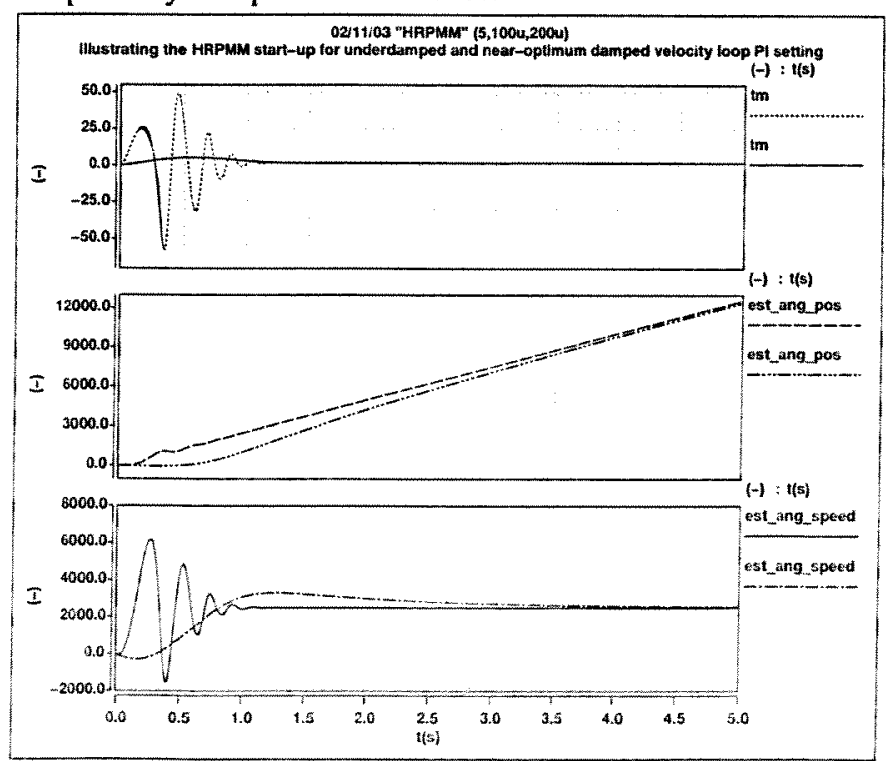

Fig. 6 Start-up in the motoring mode

Fig. 7 shows the step load response. The traces are load torque. motor torque, angular position, and speed.

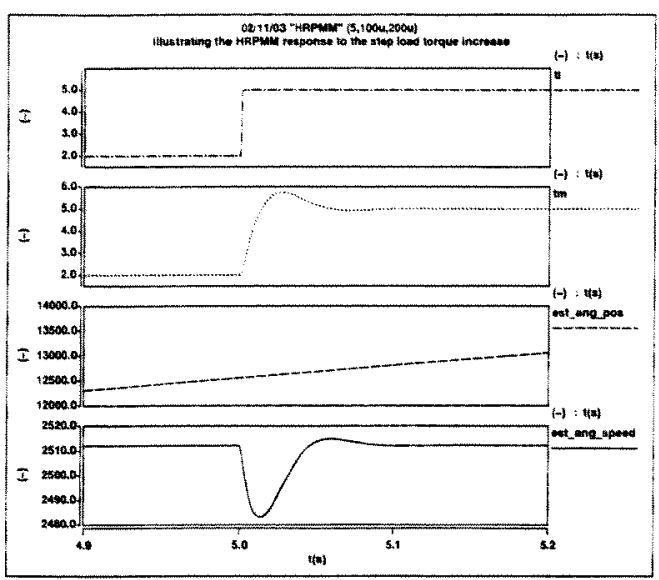

(a)

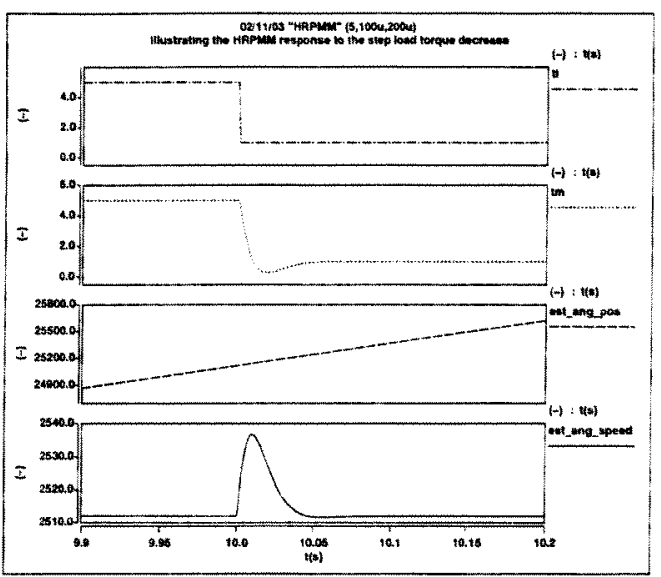

(b)

Fig. 7 Response to the step load torque (a) increase (b) decrease

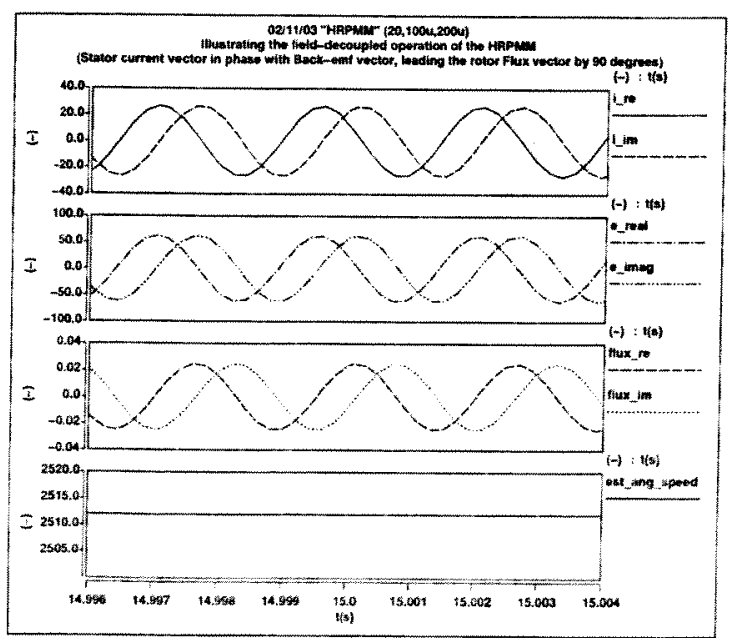

Fig. 8 Illustrating field-decoupled operation in the motoring mode

The field-decoupled operation is shown in Fig. 8. From the top down. it is seen that the stator current vector. represented in the time domain with both of its 
projections, is in phase with the back-emf vector, leading the rotor flux vector by $90^{\circ}$. Speed is shown in the bottom trace.

In the generating mode, Fig. 9 depicts a near-step response to the load current change. Shown from top to bottom are the $d c$ bus capacitor current, current drawn from the $d c$ bus, real power drawn from the generator, the shaft speed, the load current, and the $d c$ bus voltage.

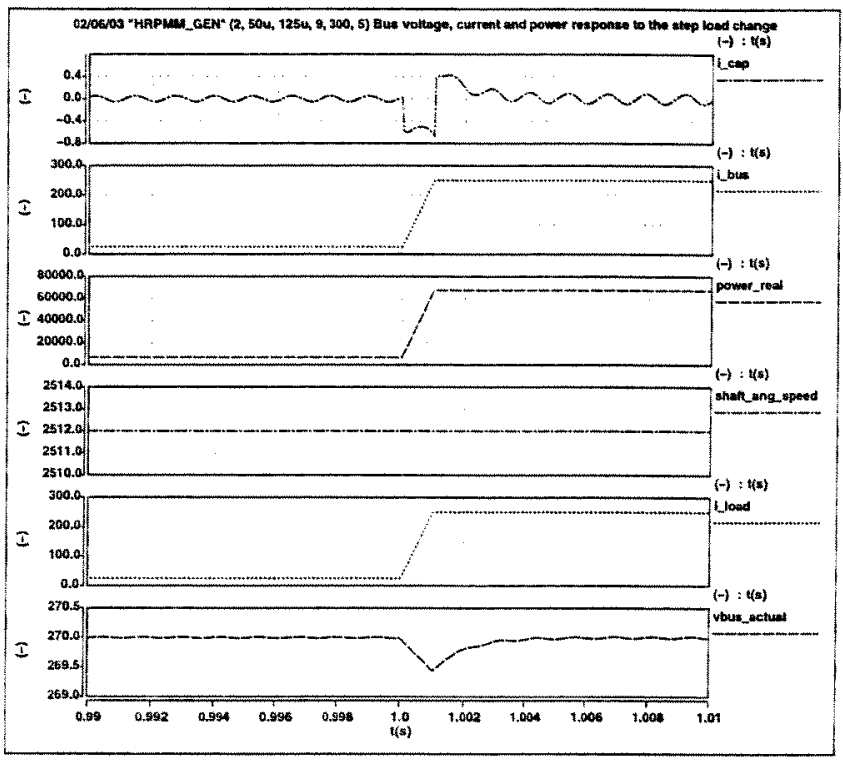

(a)

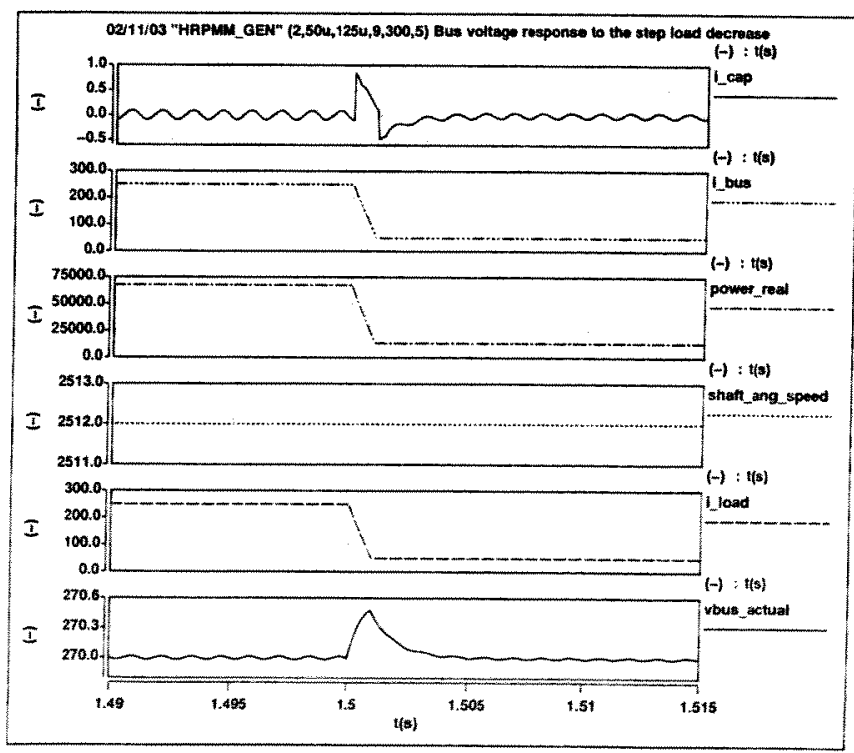

(b)

Fig. 9 Response to the load current (a) increase (b) decrease
Fig. 10 shows the response of some of the model variables to the load current increase. Shown from top to bottom in Fig. 10(a) are the time domain counterparts of stator current vector, back-emf vector, the stationary voltage vector ( $\mathbf{V}_{\text {Real,Imag }}$ in Fig. 5), followed by the machine shaft speed, load current, and dc bus voltage.

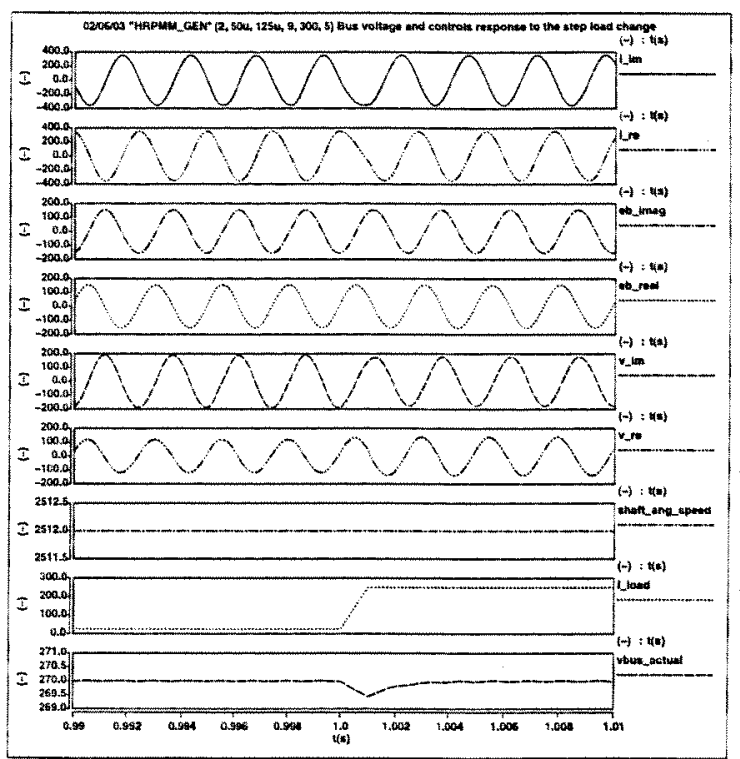

(a)

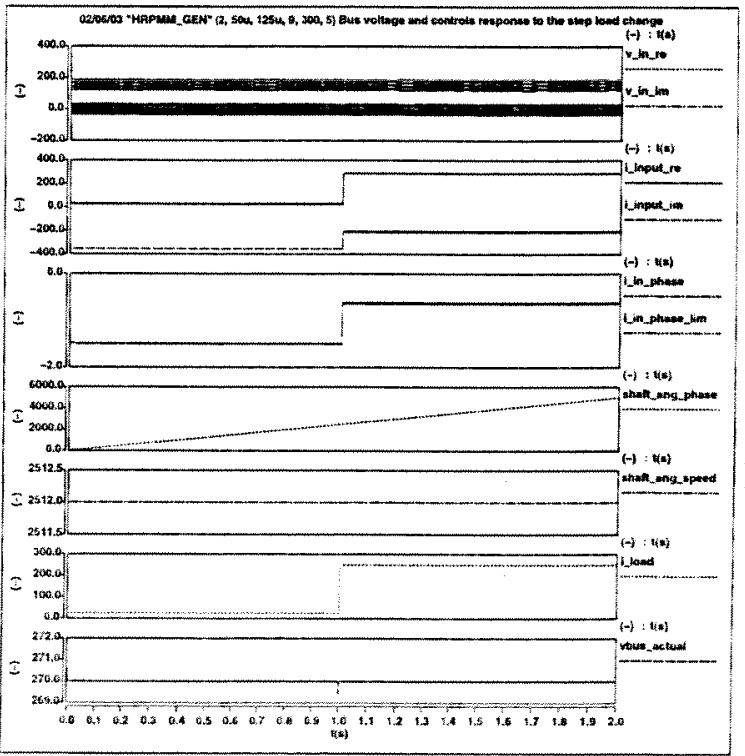

(b)

Fig. 10 Illustrating operation in the generating mode

Fig. 10(b) depicts (from top to bottom) the time domain waveforms corresponding to the following variables of Fig. 5: the voltage vector $\mathbf{V}_{\text {reim }}$, the current vector $\mathbf{I}_{\text {rref,iref, followed by the phase information for }}$ 
cos gen \& sin gen blocks, the shaft angular phase and speed, the load current, and the bus voltage.

In most of the simulations, the algorithm selection tab had to be appropriately adjusted in order for the simulation to converge to a solution. Typically, the settings were: Target Iterations $=9$ (default is 3 ), Max Newton Iterations $=300$ (default is 100 ), and Newton Step Density $=5$ (default is 1 ).

\section{CONCLUSION}

The modeling and simulation of a vector-controlled synchronous machine in motoring and generating mode has been presented. In particular, the model of the synchronous current vector to stationary voltage vector transformation incorporating the internal closing of the loops is discussed in each case, and the simulation parameters and results are shown verifying the models and demonstrating the required machine operation. The machine is controlled for a maximum torque-per-unitcurrent in the motoring mode, and for the prescribed power production in the generating mode, using mostly the same control building blocks.

\section{REFERENCES}

[1] N. Mohan et al, "Power Electronics", 2-nd Ed., John Wiley \& Sons, Inc., 1995.

[2] L. W. Matsch and J. D. Morgan, "Electromagnetic and Electromechanical Machines", 3-rd Ed., Harper \& Row, Publ., Inc., 1986

[3] P. C. Sen, "Principles of Electrical Machines and Power Electronics", John Wiley \& Sons, Inc., 1989.

[4] G. McPherson and R. D. Laramore, "An Introduction to Electrical Machines and Transformers", 2-nd Ed., John Wiley \& Sons, Inc., 1988.

[5] N. A. Losic, "Zero-Order Three-Phase Phase-Locked Loops". IEEE-lecon '03 Proceedings, Roanoke, VA, Nov. 2003, pp.29732978.

[6] S. Bouchiker et al, "Vector Control of a PM Synchronous Motor Using AC-AC Matrix Converter", IEEE Trans. on Power Electronics, Vol. 13, No. 6, November 1998, pp.1089-1099.

[7] D. Simon and D. L. Feucht, "Synchronous Motor Phase Control by Vector Addition of Induced Winding Voltages". IEEE Trans. on Industrial Electronics, Vol. 51, No. 3, June 2004, pp.537-544.

[8] W. Qian at al, "Torque Ripple Minimization in PM Synchronous Motors Using Iterative Learning Control". IEEE Trans. on Power Electronics, Vol. 19, No. 2. March 2004. pp.272-279.

[9] M. F. Rahman and L. Zhong. "Comparison of Torque Responses of the Interior Permanent Magnet MotorUnder PWM Current and Direct Torque Controls". IEEE-lecon "99 Procetdings, San Jose, CA. Nov/Dec. 1999. pp. $1+64+1+70$.
[10] L. Tang et al, "A Novel Direct Torque Controlled Interior PM Synchronous Machine Drive With Low Ripple in Flux and Torque and Fixed Switching Frequency", IEEE Trans. on Power Electronics. Vol. 19, No. 2, March 2004, pp.346-354.

[11] G. J. Su and J. W. McKeever, "Low-Cost Sensorless Control of Brushless DC Motors With Improved Speed Range". IEEE Trans. on Power Electronics, Vol. 19, No. 2, March 2004, pp.296-302.

[12] J. S. Lawler et al, "Extending the Constant Power Speed Range of the Brushless DC Motor Through Dual-Mode Inverter Control", IEEE Trans. on Power Electronics, Vol. 19, No. 3, May 2004, pp. $783-793$

[13] R. Monajemy and R. Krishnan, "Control and Dynamics of Constant Power Loss Based Operation of PM Synchronous Motor Drive System", IEEE-lecon "99 Proceedings, San Jose. CA, Nov/Dec. 1999, pp.1452-1457.

[14] N. A. Losic, "Modeling and Applications of Space Vector Pulse Width Modulator", IEEE-lecon " 99 Proceedings, San Jose, CA, Nov/Dec. 1999, pp.330-335.

[15] N. A. Losic et al, "Modeling and Design of a Vector-Controlled PWM Active Rectifier", IEEE-lecon '01 Proceedings, Denver, CO, Nov/Dec. 2001, pp.1096-1101. 